\title{
Eddy-Induced Variability in a Transatlantic Section: Argo Observing System-Gyroscope 0302 Cruise Comparison
}

\author{
Manuel Vargas-Yáñez,* Gregorio Parrilla, ${ }^{+}$Alicia lavín,\# Pedro Vélez-Belchí, $@$ \\ César González-Pola, \& and Alonso Hernández-Guerra** \\ * Instituto Español de Oceanografía, Málaga, Spain \\ + Instituto Español de Oceanografía, Madrid, Spain \\ \# Instituto Español de Oceanografía, Santander, Spain \\ @ Instituto Español de Oceanografía, Santa Cruz de Tenerife, Spain \\ \& Instituto Español de Oceanografía, Gijón, Spain \\ **Facultad de Ciencias del Mar, Universidad de Las Palmas de Gran Canaria, Las Palmas de Gran Canaria, Spain
}

(Manuscript received 10 December 2003, in final form 15 October 2004)

\begin{abstract}
Ocean hydrological sections provide a very useful mean to study the ocean circulation as well as to determine water mass properties and to estimate fluxes. One basic method for their analysis is the spatial interpolation of data, obtained from a set of predefined stations, into a regular grid for contouring isolines and for further calculations. The shortest length scales that can be solved are limited by the distance between stations. Some of these scales, though resoluble by the sampling design, may be, with respect to time variability, shorter than the time that is needed to complete the section. This situation can produce a lack of synopticity in the obtained data, which is not usually addressed in oceanographic studies because the sequential repetition of oceanographic surveys is not an easy task. Here two samplings are compared-one by CTD- and another by Array for Real-Time Geostrophic Oceanography (Argo)-type profilers-of the same zonal section with a 5-day delay. The integral time scale for the mesoscale field is around 11 days, which implies that the mesoscale signal obtained from consecutive transmissions of the profilers are weakly correlated. The mesoscale field in a transatlantic section, which typically takes 20 days to be carried out, cannot be considered as synoptic.
\end{abstract}

\section{Introduction}

The ocean circulation and its water mass distribution and variability present different length and time scales. We can distinguish length scales from the order of the basin size to mesoscale (some hundred kilometers), and from a continuum of downscaling length scales down to microturbulence. Our knowledge about the spatial distribution of the different properties of the ocean comes mainly from in situ measurements and satellite data, and more recently from autonomous profilers. One of the main techniques used in the analysis of the data from oceanographic surveys is their interpolation into a regular grid for contouring and for further calculations. This provides information about the water mass distri-

Corresponding author address: Dr. Manuel Vargas-Yáñez, Instituto Español de Oceanografía, C.O. Málaga Fuengirola, Puerto Pesquero de Fuengirola s/n, 29640 Fuengirola, Málaga, Spain. E-mail: manolo.vargas@ma.ieo.es butions as well as estimates of water, heat, salt, etc., fluxes.

Some of the most frequently used interpolation methods are the distance-weighting and optimal interpolation (OI) schemes (Thiébaux and Pedder 1987 for a review of both methods). In both methods the length scales that can be solved are limited by the separation between observations. Obviously we cannot solve for wavelengths shorter than twice the separation between stations. Weighting schemes allow the filtering out of those wavelengths in which we are not interested, or simple separation thereof (Maddox 1980; Gomis and Alonso 1990; Tintoré et al. 1991). Optimal interpolation also allows some sort of filtering through the choice of the noise-to-signal ratio and the decaying distance of the used autocovariance function (Bretherton et al. 1976; Roemmich 1983; Pedder 1989; Fukimori and Wunsch 1991; Gomis et al. 2001; Lavín 1999). The length scales that are retained in the analysis depend on which is the phenomenon of study. Mesoscale, and even submesoscale, eddies will have great importance if the 
interest is in the physical-biological coupling in the upper hundred meters (Rodríguez et al. 2001; Barton et al. 1998; McGillicuddy and Robinson 1997; Fernández and Pingree 1996). On the other hand, if the interest is in some property average, the eddy-induced variability will simply be considered as a noise.

A good example of the eddy-induced variability around a mean value is found in the historical study of oceanographic sections. One of the best sampled of these sections is the one along the $24.5^{\circ} \mathrm{N}$ parallel in the Atlantic, from the African coast to the Bahamas, which has been sampled in 1957, 1981, 1992, and 1998 (Fuglister 1960; Roemmich and Wunsch 1984, 1985; Parrilla et al. 1994; Bryden et al. 1996; Lavín et al. 2003; Baringer and Mollinari 1999). In these works it has been shown that there is a warming in the intermediate layer (800$3000 \mathrm{~m}$ ), which is represented by a trend in the potential temperature mean value (zonally averaged). The temperature change is obscured by the shorter timescale variability that is induced by the eddy field; therefore, it was filtered using a Gaussian filter (Roemmich and Wunsch 1984; Parrilla et al. 1994). This eddyinduced noise also imposes a limitation to the significance of the temperature trends that are detected from 1957 to 1992.

It is usually assumed that the dataset obtained in an oceanographic survey is synoptic, independently of which the interpolation method is used, and the length scale of the phenomenon is of interest. Distributions of temperature, salinity, etc., usually exhibit strong eddy activity (Fig. 2 in Bryden et al. 1996 or Fig. 5 in Lavín et al. 2003), with the separation between stations or, as already mentioned, a particular interest being the only limitation for their resolution. Nevertheless, these structures can have time scales that are shorter than the time needed to accomplish the complete sampling. In this case the mesoscale field left after interpolation, and contouring would not give any real picture of the spatial domain covered by the survey. However, this eddy field can still be representative of certain statistical properties of the real one, such as the main length scales or the variance of the field.

The problem of determining whether or not a certain dataset can be considered synoptic is difficult, because oceanographic surveys are not frequently repeated. One exception can be seen in Gomis et al. (2001), where an oceanographic survey was repeated 4 times in a 14- day period and a synopticity test for each survey could be addressed. The area that was covered in that case was quite reduced $(100 \mathrm{~km} \times 80 \mathrm{~km})$. A similar test, using traditional sampling techniques, is very difficult for large areas of the ocean.

In March 2002, the Instituto Español de Ocean- ografía (IEO) deployed (within the European project Gyroscope) 19 autonomous profilers in the Canary Basin. During the deployment, 38 CTD profiles were collected. One of the cruise legs was along $24.5^{\circ} \mathrm{N}$, covering the eastern half of the section that was already sampled in 1957, 1981, 1992, and 1998. The temperature and salinity fields that are obtained from the CTD casts, and the first data transmitted by the profiling floats with a 5-day delay, provided an exceptional opportunity to check the time variability of a hydrologic transatlantic section. The main objective of this paper is to show that mesoscale structures can change in a 5-day period. Taking into account that this time is shorter than the time that is needed to accomplish a transatlantic section, it is implied that the data cannot be considered as synoptic. The direct comparison of temperature and salinity profiles obtained with a CTD probe and profiling floats at the same location shows that the differences observed are a result of the high temporal variability of the mesoscale field. This result also implies that it is not possible to compare different instruments, such as CTD probes and profiling floats, at isobaric levels if there is a time delay between both datasets. As a secondary objective it is shown that the CTD-profiler comparison should be made by analyzing the $\theta-S$ relationship that is obtained for the water masses sampled with the CTD probe and the profilers.

The paper is organized as follows. Section 2 presents the dataset. Section 3 contains a comparison of the two repetitions of the $24.5^{\circ} \mathrm{N}$ section and the direct comparison of the potential temperature and salinity profiles obtained at the same locations. Section 4 deals with the estimation of the mesoscale time variability using the time series provided by the profiling floats. In section 5 the performance of the profiling floats and the CTD probe are compared, considering the $\theta-S$ diagrams that are obtained from both datasets. A summary and conclusions are given in section 6 .

\section{Data}

The cruise Gyroscope E 03-2002 (Grupo GyroscopeEspaña 2002), carried out by IEO in March 2002 within the homonymous European project, was planned to deploy 20 autonomous profilers, equipped with pressure, temperature, and conductivity sensors, along a box bounded by the parallels between $24.5^{\circ}$ and $30^{\circ} \mathrm{N}$ and the meridians between $18^{\circ}$ and $42^{\circ} \mathrm{W}$. The southern leg of the box ran from the African coast to the MidAtlantic Ridge. It coincides with the eastern half of the sections that were previously sampled in 1957, 1981, 1992, and 1998.

The profiler set consisted of 15 PROVOR and 5 


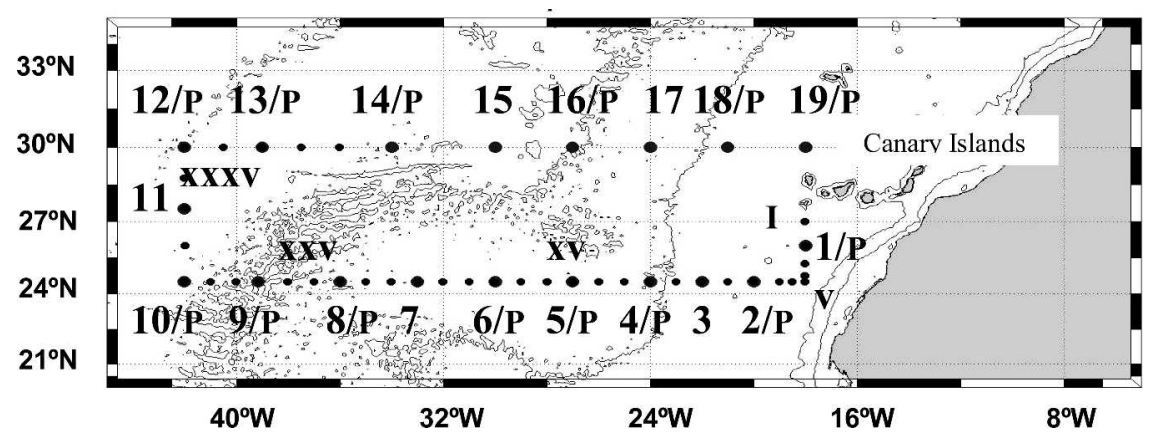

FIG. 1. Cruise map. Small dots indicate the positions of the CTD casts and large dots are the positions where autonomous profilers were deployed. CTD casts were also carried out in such locations. Profilers are labeled with numbers from 1 to 19 and CTD casts are labeled with roman numbers. The letter $\mathrm{P}$ is added to identify PROVOR profilers.

autonomous profiler explorer (APEX) types. The main difference is that the latter ones are equipped with a salinity module, providing salinity measurements directly, instead of conductivity. Both types of profilers are programed to drift at a $1500-\mathrm{m}$ depth and sink to a 2000-m depth and emerge to the surface recording a CTD profile every 10 days. One of the PROVOR profilers failed because of a programming error, so the final set was of 19 profilers (Fig. 1).

Additionally, it was also planned to make CTD stations along the box. Unfortunately, the loss of the probe limited the planned number of casts to 38 (Fig. 1). The CTD probe was equipped with redundant conductivity and temperature sensors. Water samples were also obtained for salinity calibration and oxygen and nutrient determination. The redundant sensors and water samples were used to calibrate the CTD to World Ocean Circulation Experiment (WOCE) standards (see Grupo Gyroscope-España 2002 for technical details).

The only CTD section completed was that along $24.5^{\circ} \mathrm{N}$, the one on which we will focus in section 3 . Nevertheless we will also use CTD and profiler data along the western boundary of the box and the northern section (uncompleted) for comparison of the CTD and profiler data (section 5).

The CTD was lowered to the bottom depth, which varies between some $3300 \mathrm{~m}$ near the African slope and over the Mid-Atlantic Ridge, and more than $5600 \mathrm{~m}$ in the abyssal plane. CTD profiles along the $24.5^{\circ} \mathrm{N}$ section were taken from 4 to15 March 2002. The profiles from the first section were completed by the autonomous profilers, which correspond to 8-20 March 2002. The central time point in the CTD section is 1200 UTC 9 March 2002, and that in the profiler section is 14 March 2002. There is a delay close to 5 days between both of them. If there were differences in the length scales that the station separation would be able to solve, it would indicate that mesoscale structures can evolve in a 5-day period, which is shorter than the time needed to complete the whole section-12 days for the CTD and 13 for the profiler section. Note that the three previous cruises completed the section from the African coast to the Bahamas in 23, 24, and 26 days, respectively. Because the complete section has approximately double the length of that of the section of the Gyroscope cruise, the time needed to sample the sections is similar. If a 12-day period is too long for ignoring the time evolution of mesoscale structures, the situation is even worse for the 24-day-long sections.

\section{CTD and profiler section comparison}

The CTD casts along the $24.5^{\circ} \mathrm{N}$ section (4-15 March), and the first transmission of the profiling floats (8-20 March) allow for the study of the evolution of the hydrological structures during this time period. Temperature and salinity data from the CTD and profilers were interpolated into a common grid extending from $18^{\circ}$ to $42^{\circ} \mathrm{W}$ for the upper $1000 \mathrm{~m}$. The horizontal and vertical resolutions are $25 \mathrm{~km}$ and $25 \mathrm{~m}$, respectively. The interpolation method was the same for both datasets. A background field was estimated fitting a second-order polynomial. Increments or deviations from the background field were optimally interpolated into the grid. The covariance function and the noise variance that were estimated from the temperature and salinity increment fields were

$$
\begin{aligned}
& \gamma_{\theta}(\mathbf{u}, \boldsymbol{v})=0.22 e^{-\left(\frac{\Delta x^{2}}{2 D_{x}^{2}}+\frac{\Delta y^{2}}{2 D_{y}^{2}}\right)}+0.1 \delta_{r, s}, \\
& \gamma_{S}(\mathbf{u}, \boldsymbol{v})=0.02 e^{-\left(\frac{\Delta x^{2}}{2 D_{x}^{2}}+\frac{\Delta y^{2}}{2 D_{y}^{2}}\right)}+0.005 \delta_{r, s},
\end{aligned}
$$

where $D_{x}=250 \mathrm{~km}$ and $D_{y}=33 \mathrm{~m} ; \mathbf{u}, \boldsymbol{v}$ are any couple of position vectors within the vertical section. 

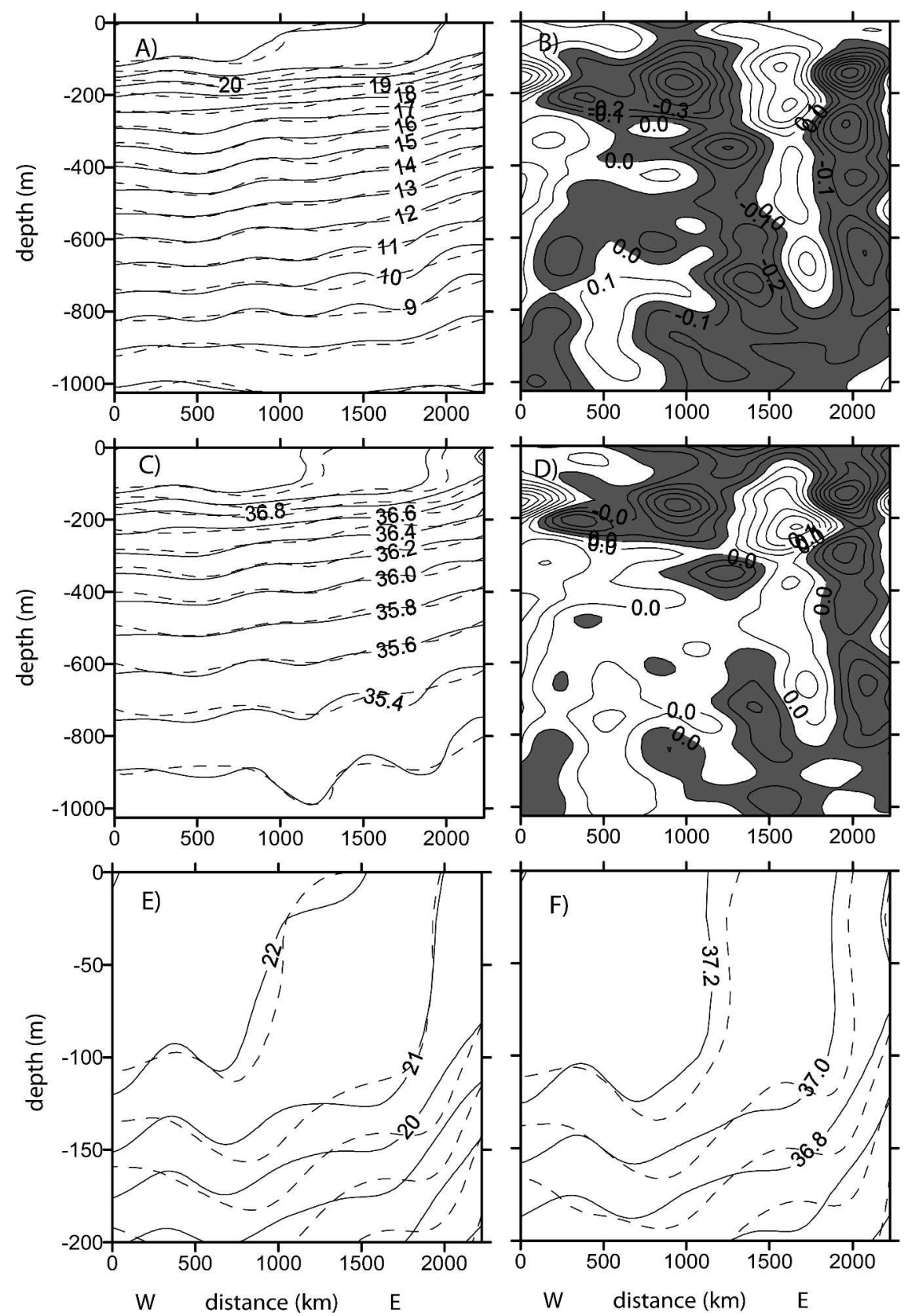

FIG. 2. (a) CTD (solid line) and profiler (dashed line) temperature sections interpolated into a regular grid with an OI scheme. (b) Differences between the interpolated fields in (a). Negative differences have been shaded for the clarity of the plot. Contour lines are each $0.1^{\circ} \mathrm{C}$. (c), (d) Same as in (a) and (b), but for the salinity field. Contour lines in Fig. 2d are each 0.025. (e), (f) An enlargement of the upper $200 \mathrm{~m}$ to highlight differences in the uppermost layer where the eddy activity is more intense.

Figures $2 \mathrm{a}$ and $2 \mathrm{c}$ show the potential temperature and salinity fields plotted from the CTD data (solid line) and from the first transmission of the profiling floats (dashed line). Figures $2 \mathrm{~b}$ and $2 \mathrm{~d}$ show the differences between both datasets. These differences range between $-0.7^{\circ}$ and $0.7^{\circ} \mathrm{C}$ for potential temperature and from -0.14 to 0.15 for salinity. The background fields (not shown), which capture those structures with length scales of the order of the analyzed section, are almost identical for both datasets. Differences seem to be associated with the different shape and position of the mesoscale structures. To enhance these differ- 
ences we present a zoom of the upper $200 \mathrm{~m}$ (Figs. 2e,f).

Nevertheless, before accepting that the differences observed are a result of the time variability of the eddy field, we have to discard other causes. CTD casts have a separation of some $100 \mathrm{~km}$; meanwhile, the profiling floats are separated around $300 \mathrm{~km}$. The covariance function that is used for interpolation [Fig. 3a and expression (1)] implies that the increment field has no energy at wavelengths shorter than $600 \mathrm{~km}$. Nevertheless, the OI scheme is applied to datasets with different spatial resolutions, and to our knowledge the spectral response of this interpolation method is not clearly established. If the CTD-interpolated field would contain wavelengths that are shorter than the field interpolated from the profiler data, it could cause the observed differences. To check this matter, we calculated the Fourier coefficients for the CTD- and profiler-interpolated fields of increments or residuals:

$$
C_{k, l}=\frac{1}{N_{x} N_{y}} \sum_{j} \sum_{i} \theta_{i, j} e^{-j 2 \pi\left(\frac{k}{L_{x}} x_{j}+\frac{l}{L_{y}} y_{i}\right)},
$$

where $\theta$ is the interpolated increment field $j, i$ denotes the horizontal and vertical positions within the grid, $N_{x}$ and $N_{y}$ are the number of grid points on the horizontal and vertical directions, and $L_{x}$ and $L_{y}$ the horizontal and vertical lengths of the grid. Each plane wave has a wavenumber $\mathbf{k}=2 \pi\left[\left(k / L_{x}\right),\left(l / L_{y}\right)\right]$ and wavelength $\lambda_{\mathbf{k}}=2 \pi /|\mathbf{k}|$. The averaged power of the signal corresponding to each wave is $\left|C_{k, l}\right|^{2}$. Figure $3 \mathrm{~b}$ shows the power associated with each plane wave as a function of $1 / \lambda$ for the interpolated temperature field. Figure $3 c$ is the same but selects only the horizontal plane waves, that is, those with $l=0$ [see expression (2)], because it is on the horizontal direction where both datasets have a different resolution. We see that in both cases the power of the signal is limited to values of $1 / \lambda$ lower than $0.0018 \mathrm{~km}^{-1}$, that is, there is no energy associated with the wavelengths shorter than $550 \mathrm{~km}$. This first result indicates that the differences outlined in Fig. 2 are not a result of the different spatial resolution of both datasets.

Observed differences could also be a result of the interpolation error. The OI allows the estimation of the error variance, defined as $\left\langle\left(\theta_{\mathbf{g}}-\hat{\theta}_{\mathbf{g}}\right)^{2}\right\rangle$, where $\theta_{\mathbf{g}}$ is the true value at the grid point $\mathbf{g}, \hat{\theta}_{\mathbf{g}}$ is the estimated value, and the brackets denote expectation. The error variance is estimated at each grid point. Taking the square root of the error variance and averaging over the whole section we get an average standard deviation for the error of $0.14^{\circ} \mathrm{C}$ for temperature and 0.03 for salinity. Values as large as $0.7^{\circ} \mathrm{C}$ or 0.14 are not likely to
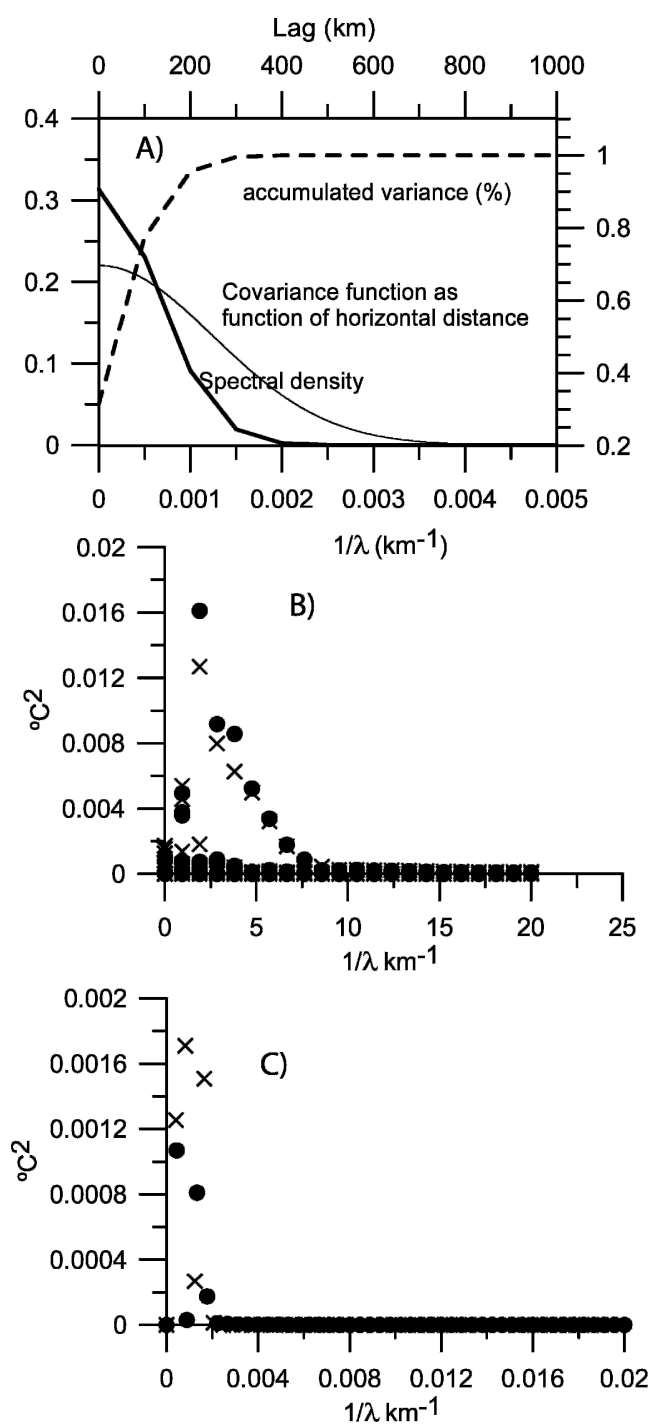

FIG. 3. (a) Thin solid line is the temperature autocovariance function used in the OI scheme as a function of the horizontal coordinate [making $\Delta y=0$ in expression (1), see text]. Thick line is the spectral density using the Fourier transform of the autocorrelation function, and the dashed line is the accumulated variance, obtained by integration of the spectral density. (b) Spectral analysis using a two-dimensional Fourier decomposition of the CTD optimally interpolated field (crosses) and profiler field (filled circles). (c) Same as (b), but considering only horizontal waves $(l=0)$. In (a)-(c) we express the power associated to each wave as a function of the inverse of the wavelength.

be caused by the interpolation error, so we discard this cause.

The contouring of interpolated fields evidences variations in the mesoscale structures. For instance, Figs. $2 \mathrm{e}$ and $2 \mathrm{f}$ show that the position where the $22^{\circ} \mathrm{C}$ isotherm and the 37.2 isohaline outcrop the surface has shifted more than $100 \mathrm{~km}$ from one survey to the other. Nevertheless, another possibility is that all of the dif- 
ferences outlined along this section are an artifact of the analysis and interpolation process. The interpolated sections are simply smoothed versions of the coincident CTD and profiler measurements, and a direct comparison of these profiles should show these differences too. To perform this analysis we only use the PROVOR floats for which there are 10 coincident with CTD casts (only three APEX profilers are coincident). The differences between temperature and salinity profiles at coincident locations in the upper 1000-m range between $-1^{\circ}$ and $1^{\circ} \mathrm{C}$ and -0.5 and 0.2 , respectively. As in the interpolation process the existence of errors in the observations is assumed, the interpolation process smoothes the field as is already evidenced by the spectral response of the interpolated fields (Figs. 3b,c). This explains that the differences are slightly larger in the direct comparison.

The mesoscale field, estimated from the interpolated sections, has a standard deviation that changes along the water column, with the maximum in the upper 200 $\mathrm{m}$ where the eddy activity is likely to be more intense. Averaging over the water column, we get the values of $0.18^{\circ} \mathrm{C}$ and 0.03 for the temperature and the salinity, respectively. If we estimate the standard deviation for the mesoscale field using the PROVOR profiles (once a second-order polynomial is subtracted to account for the background or large-scale field), we get the values of $0.21^{\circ} \mathrm{C}$ and 0.04 for the temperature and the salinity, respectively. As already stated, the differences between the CTD and profiler data range between $-1^{\circ}$ and $1^{\circ} \mathrm{C}$. The standard deviations of these differences are $0.3^{\circ} \mathrm{C}$ and 0.05 for the temperature and salinity. Note that the standard deviation of the difference between the CTD and profilers is $\sqrt{\sigma_{\mathrm{CTD}}^{2}+\sigma_{P}^{2}-\gamma(\mathrm{CTD}, P)}$, where $\sigma_{\text {CTD }}^{2}$ is the variance of the CTD field and $\sigma_{P}^{2}$ is the variance of the profiler data, which we assume is the same. Here, $\gamma$ is the covariance between the CTD and profiler data. Both datasets must be correlated because they are only 5 days apart (we will see the time scale of the mesoscale field in the following section). Note that for the profiler data the standard deviation is 0.21 , which, multiplied by $\sqrt{2}$, is 0.3 , the standard deviation of the difference. As mentioned above, the standard deviation of the differences should be lower because of the correlation of both datasets; nevertheless, this can be a result of errors in the estimation because of the low number of profiles used for comparison (10 profiles). These simple calculations show that the differences between the CTD and profiler sections are coherent with the range of variability that is associated with the mesoscale field. These differences are several orders of magnitude higher than the instrument accuracy, so it cannot be the cause of the differences. The differences also cannot be attributed to the different resolution of both datasets. These results, together with the visual inspection of the contoured fields (Fig. 2), suggest that the differences that are observed are a result of the mesoscale variability. Mesoscale features can change significantly in a 5-day period, which is shorter than the time that is needed to complete a transatlantic section. This result shows that there is a lack of synopticity in these sections.

\section{Autocorrelation and integral time scale}

In the previous section it has been shown that the mesoscale field can change in a time, which is comparable to that needed to complete half a transatlantic section (in our case, around 12 days). Nevertheless some of the statistics that are used have been derived from a single CTD survey, which is usually the procedure in oceanography. To estimate the covariance between two stations separated a certain distance, let us say $d$, we search all of the pairs of measurements that are separated by $d \pm b / 2$, where $b$ is the size of the bin. This implies that the statistics of the signal are homogeneous, and we substitute averages over time by averages over a certain spatial domain. Most of the OI methods were initially developed by meteorologists. In meteorology, long time series at different fixed stations are available; the covariance between them can be obtained and we can use it in the OI schemes. Contrary to this situation, in oceanography the background field and the statistics of the signal are frequently estimated from one single oceanographic survey. The use of autonomous profilers can produce more accurate estimations of the signal statistics. Nevertheless, this method still has certain limitations when compared with meteorological stations, because profilers drift and it is more difficult to obtain the correlation between two different locations or the autocorrelation for a single site.

Figure 4a shows the time evolution of potential temperature at four different depths for the profiler 4 , around $24.5^{\circ} \mathrm{N}$ and $24^{\circ} \mathrm{W}$. The time series extend from the deployment at mid-March to mid-October; time is expressed in years. We can see a low-frequency variability clearly, and a higher variability superimposed on it. We also have to take into account that the profiler drifted from its initial position at $24.5^{\circ} \mathrm{N}, 24^{\circ} \mathrm{W}$ to $25^{\circ} \mathrm{N}$, $26^{\circ} \mathrm{W}$. The shape of the low-frequency curve looks like a typical seasonal cycle, with minimum temperatures in late winter and maximum ones in late summer in the upper layer $(10,50 \mathrm{~m})$ and a certain delay at deeper layers $(100 \mathrm{~m})$. The seasonal cycle should be similar for the initial and final position of the profiler; thus, we could simply fit a harmonic with a 1 -yr period to the time series to remove the low-frequency variability, and 

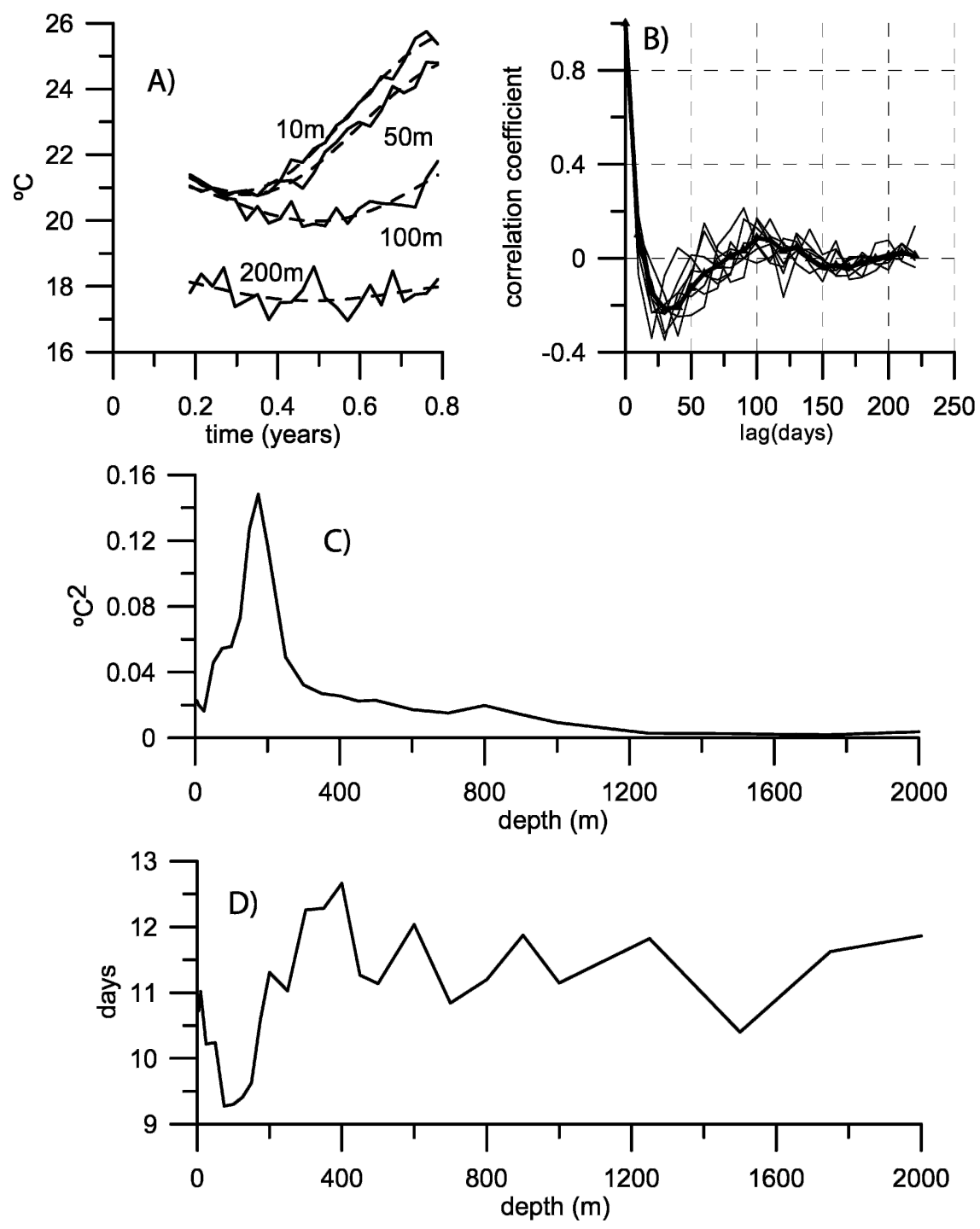

FIG. 4. (a) Time series of potential temperature from profiler 4 (solid lines). Though the analysis was done for 25 depths from the surface to $2000 \mathrm{~m}$, here we only include four series for clarity of the plot. Dashed lines are the least squares fit using expression (3) in the text. (b) Autocorrelation function for some selected depths for the residual time series obtained from the fit (thin solid line). We plot only some of the 25 series analyzed for clarity. The thick line is the averaged autocorrelation function. (c) Variance of the residuals (eddy field) as a function of the depth; and (d) integral time scale as a function of the depth.

consider that the residuals constitute the mesoscale signal. If the drifting of the profiler was random around the initial position, we could estimate the signal statistics of these fluctuations without considering any differences between those residuals that are caused by changes in the temperature field of the initial position as a result of the passing of eddies (strictly speaking time variability) or changes in the potential temperature that is measured by the profiler as a result of its change in position (spatial variability). The sequence of positions occupied by the profiler shows a continuous drift to the northwest. If the background field was homogeneous or if its variability could be neglected over the distance traveled by the profiler, we could, once again, limit the analysis of the low-frequency variability to the subtraction of the seasonal cycle. As mentioned above, the initial and final position differ in $2^{\circ}$ longitude and $1 / 2^{\circ}$ latitude; therefore, we included a linear trend in the low-frequency variability fit to reflect the changes in the background temperature field. Finally, 
we used the following function to model the seasonal cycle and the possible drift effect over the low-frequency variability:

$$
a+b t+c \cos (2 \pi t)+d \sin 2(\pi t) .
$$

For profiler 4 we fit (3) to 25 time series corresponding to 25 depths from the surface to $2000 \mathrm{~m}$. The fit is represented in Fig. 4a by the dashed lines. Because profiler 4 was deployed close to the eastern boundary of the section, a similar analysis was applied to profiler 10, in the western edge of it, and no differences were found in the following results.

Once the low-frequency variability was subtracted at the 25 depths selected, the autocovariance function for the residuals that represent the mesoscale field was estimated. Figure $4 \mathrm{~b}$ shows the autocorrelation function at 5-, 50-, 100-, 250-, 500-, 1000-, 1500-, and 2000-m depths. We only represent these eight depths for clarity; nevertheless, the thick line represents the average of the autocorrelation function for the 25 depths that are analyzed. The lag is expressed in days with an interval of 10 days, which is the time between two consecutive transmissions. A striking result is that the correlation for a 10-day delay is quite low. To obtain another estimation of the decorrelation between hydrological profiles that are delayed in time, we estimated the correlation between the PROVOR temperature profiles over the $24.5^{\circ} \mathrm{N}$ section and the coincident CTD profiles (after detrending). The correlation obtained was 0.8. PROVOR and CTD profiles were delayed 3 days (APEX profiles were delayed 9 days, which were responsible for the average 5-day delay). Using the profiler time series, we obtained a correlation of 0.1 for a 10-day lag. Interpolating linearly from 1 (at $0 \mathrm{lag}$ ) to 0.1 (at 10-day lag), we estimate a value of 0.73 for a 3 -day lag, which seems to be coherent with the 0.8 value obtained from the direct comparison of profiler and CTD data.

To define the time scale of a phenomenon the integral time scale is widely used, which is defined as

$$
\tau=\int_{0}^{\infty} \rho(s) d s,
$$

where $(\rho s)$ is the correlation coefficient for the lag $s$. In practice, the estimated correlation function decays to zero and then remains close to zero, but with positive and negative oscillations that make its estimation uncertain. Freeland (1990) solves this problem by fitting an exponential function to the autocorrelation function that is estimated for the first lags. Lenschow and Stankov (1986) calculated $\tau(r)=\int_{0}^{r} \rho(s) d s$, and then took the first maximum of the function $\tau(r)$. Figure $4 d$ shows the integral time scale that is calculated following
Lenschow and Stankov (1986) for each of the 25 depths studied. We also computed the integral time scale considering only the autocorrelation function until its first zero and no main changes were observed. The average value is 11 days.

To check the coherence of this result we consider that the length scale is related to the time scale by the mean speed of the flow, which can range between 10 and $30 \mathrm{~cm} \mathrm{~s}^{-1}$ for the eastern boundary and between 5 and $10 \mathrm{~cm} \mathrm{~s}^{-1}$ for the rest of the section (Lavín et al. 2003). For these values, the mesoscale length scale would range between 100-300 and 50-100 km respectively, coinciding in a rough way with an spectral analysis that is applied to the nonsmoothed CTD data (not shown), as well as with previous works (Lavín 1999).

According to these results, two consecutive transmissions of a profiler (10 days apart) are very weakly correlated (concerning the mesoscale field). But what is more interesting follows: if we need 12 days to complete the section, the mesoscale field that is obtained at the beginning and the end of the section is very poorly correlated and synopticity is not a good hypothesis. The situation would be even worse when comparing the eastern and western North Atlantic basins in those surveys that completed the section from Africa to the Bahamas.

\section{Influences on the intercomparison of CTD and profiler performances}

One of the objectives of the Gyroscope E 03-2002 cruise was to compare the performance of the profilers. Because there is a small delay (5 days) between both sections it is implied that the eddy variability is the main source of error as it happened in other cases, for example, the warming detection from 1957 to 1992 (Parrilla et al. 1994). The temperature sensors used in the profilers seem to perform properly, but the same could not be said of the conductivity sensors (Wong et al. 2003). It is clear that the eddy activity can produce large vertical excursions of isopycnals. Roemmich and Wunsch (1985) stated that a single eddy could change the temperature at a $1000-\mathrm{m}$ depth by $2^{\circ} \mathrm{C}$ over a distance of $200 \mathrm{~km}$. It is clear that it is not sensible to compare temperature or salinity at isobaric levels. On the other hand, if the effect of eddy activity is just to rise (cyclonic eddies) or sink (anticyclonic) the isopycnals, the $\theta-S$ relationships would remain constant. Figure 5a shows the $\theta-S$ diagrams for both the CTD casts and the first transmission of the profilers. Because there are two different types of profilers and each one has a different type of salinity sensor, we used only the PROVOR profilers (14), because a statistical analysis would be more difficult with APEX profilers (only 5). 

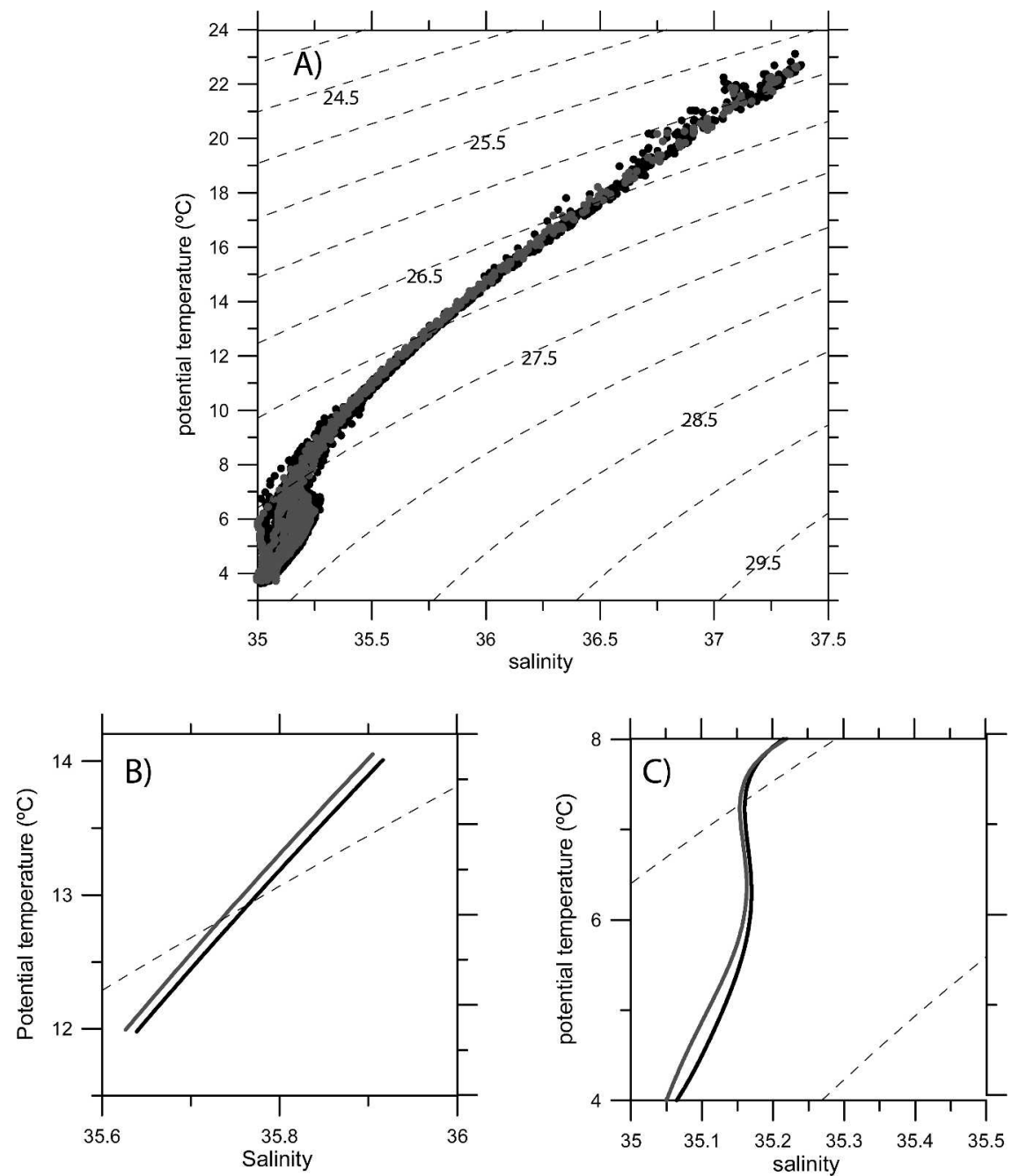

FIG. 5. (a) The $\theta-S$ diagrams for the first transmission of PROVOR profilers 1-13 (light dots) and the respective CTD casts (dark dots). (b) Detail of the $\theta-S$ fit for both the profiler (light curve) and CTD (dark curve) data using a third-order polynomial for the main thermocline $\left(9^{\circ}-22^{\circ} \mathrm{C}\right)$, and (c) the same for the intermediate layer $\left(4^{\circ}-8^{\circ} \mathrm{C}\right)$.

For comparison we used only those CTD casts at the same locations as the PROVOR profilers, that is, profilers $1-13$ and the CTD casts coinciding with them.

As noted in previous sections, it is clear that the direct comparison of two coincident profiles makes no sense because of eddy variability. Nevertheless, because the total length of the spatial domain is much larger than the eddy length scale, fluctuations should be canceled out and the average $\theta-S$ relationship should be the same, obviously within the uncertainty imposed by the noise dispersion, which is a result of eddy variability.

Apparently, from Fig. 5a, the salinity values corresponding to the PROVOR profilers are slightly displaced toward lower values than those of the CTD. The mean $\theta-S$ curve has to be modeled by fitting a smooth function to the cloud of $\theta-S$ points. Another possibility is to average the salinity for each temperature value.

Figures $5 \mathrm{~b}$ and $5 \mathrm{c}$ show the result of fitting a thirdand fourth-order polynomial to the main thermocline $\left(9^{\circ}-22^{\circ} \mathrm{C}\right)$ and the intermediate $\left(4^{\circ}-8^{\circ} \mathrm{C}\right)$ layers. In the fitted polynomial the salinity is considered as a function of potential temperature, which is assumed to have no error. Though the fitted line is displaced to lower salinities for the profiler data, the differences in the parameters of the fit were not significantly different. This does not allow for the rejection of the null hypothesis that both the profiler and CTD give the same $\theta-S$ relation. Another factor to bear in mind is that the length of the section is $2200 \mathrm{~km}$ in the horizontal, which is around 7 times the considered mesoscale length scale of $300 \mathrm{~km}$ (Lavín 1999). This makes it easy to find differ- 
ences in the mean values or in the least squares fit. Another factor to be considered is that confidence intervals were calculated using the usual expressions (see, i.e., Jenkins and Watts 1968, their appendix A4.1), where the autocorrelation of the residuals is not taken into account. The autocorrelation of residuals reduces the degrees of freedom and enlarges the confidence intervals, making it more difficult to reject the null hypothesis. This problem was faced by Roemmich and Wunsch (1984), Parrilla et al. (1994), and Lavín et al. (2003), considering that the number of degrees of freedom is the number of length scales (for the mesoscale) contained in the complete length of the section. This procedure was applied to the isobaric levels where the correlation between consecutive casts was considered. In the least squares fit the situation is not so simple. Two consecutive values of temperature in the cloud of points from Fig. 5a can correspond to nonconsecutive stations and different isobaric levels. For this reason we ignore the autocorrelation of residuals and simply call the attention of the reader to this point. The two factors discussed above support the fact that the differences found in both datasets are compatible with the eddy variability. Contrary to this, we notice that the displacement toward low salinity is observed in the whole water column, in particular in the main thermocline and the intermediate layer.

\section{Summary and conclusions}

Transatlantic sections typically need around 20 days to be completed. The contouring of different properties over these sections show an intense mesoscale activity with length scales of the order of $100 \mathrm{~km}$. The study of the time variability of these structures is not usually addressed, because the repetition in time of these sections is difficult. For this reason it is not usually checked whether or not the datasets obtained in these sections are synoptic. In other words, the mesoscale structures that are sampled at the beginning of the section could have evolved when similar structures are sampled at the end of it. If this were the case, the contoured mesoscale field would not represent a real picture of the section at any time. The Gyroscope 03-2002 cruise was a unique opportunity to obtain two repetitions of such a section within around a 5-day delay. The analysis of two independent datasets that are obtained from the CTD survey and the first transmission of the profilers shows that the mesoscale structures can change in time during the time that is needed to complete the section. The analysis of the interpolated fields and the direct comparison of temperature and salinity profiles obtained at the same locations with some days of delay show differences that cannot be attributed to interpolation errors or the different resolution of both datasets. The range of these differences is coherent with the range of variability of the eddy field. According to our results, the eddy field has an integral time scale of 11 days. Consecutive transmissions of the profilers (10 days apart) are very weakly correlated, and the mesoscale field of the initial and final parts of a transatlantic section cannot be considered as synoptic. The mesoscale field that is typically contoured in this kind of section could represent the statistical properties of the true field (length scales, variance, etc.), but it would not represent any real situation. This would not be very important if we were interested in some average property, because in that case the mesoscale field should be removed, but we think that other studies, for instance, those related to the physical-biological coupling, could be seriously affected by this lack of synopticity.

Our results show that it is not possible to compare temperature and salinity fields that are obtained with different instruments if there is a certain time delay. For instance, for a 3-day delay, the temperature field is decorrelated from 1 to $0.7-0.8$, and differences over the isobaric levels range between $-1^{\circ}$ and $1^{\circ} \mathrm{C}$, which is several orders of magnitude higher than the accuracy of the instruments $\left(0.002^{\circ} \mathrm{C}\right)$. Instead of this, we propose that the $\theta-S$ properties of the water masses that are sampled should be compared. The average $\theta-S$ obtained with different instruments can be compared, and the time variability imposed by the eddy field could be removed.

Acknowledgments. We thank Norbert Cortes and David Marcote who deployed the profilers and the crew of Vizconde de Eza for their invaluable help during the whole cruise. We also thank Nicolás González, José Escánez, Rosario Carballo, Gerardo Casas, Eugenio Fraile, Venicio Pita, Elena Tel, and Daura Vega who contributed greatly to the acquisition and control of the data. This research has been funded by the European Commission project Gyroscope (EVK2-CT2000-00087).

\section{REFERENCES}

Baringer, M. O., and R. Mollinari, 1999: Atlantic Ocean baroclinic heat flux at 24 to $26^{\circ}$ N. Geophys. Res. Lett., 26, 353-356.

Barton, E. D., and Coauthors, 1998: The transition zone of the Canary Current upwelling region. Progress in Oceanography, Vol. 41, Pergamon, 455-504.

Bretherton, F. P., R. E. Davis, and C. B. Fandry, 1976: A technique for objective analysis and design of oceanographic experiments applied to MODE-73. Deep-Sea Res., 23, 559-582.

Bryden, H., M. J. Griffiths, A. Lavín, R. C. Millard, G. Parrilla, and W. M. Smethie, 1996: Decadal changes in water mass 
characteristics at $24^{\circ} \mathrm{N}$ in the subtropical North Atlantic Ocean. J. Climate, 9, 3162-3186.

Fernández, E., and R. D. Pingree, 1996: Coupling between physical and biological fields in the North Atlantic subtropical front southeast of the Azores. Deep-Sea Res., 43A, 13691393.

Freeland, H. J., 1990: Sea surface temperatures along the coast of British Columbia: Regional evidence for a warming trend. Can. J. Fish. Aquat. Sci., 47, 346-350.

Fuglister, F. C., 1960: Atlantic Ocean Atlas of Temperature and Salinity Profiles and Data from the International Geophysical Year of 1957-1958. Atlas Series, Vol. 1, Woods Hole Oceanographic Institution, $209 \mathrm{pp}$.

Fukimori, I., and C. Wunsch, 1991: Efficient representation of the North Atlantic hydrographic and chemical distributions. Progress in Oceanography, Vol. 27, Pergamon, 111-195.

Gomis, D., and S. Alonso, 1990: Diagnosis of a cyclogenetic event in the western Mediterranean using an objective technique for scale separation. Mon. Wea. Rev., 118, 723-736.

— - S. Ruíz, and M. A. Pedder, 2001: Diagnostic of the 3D ageostrophic circulation from a multivariate spatial interpolation of CTD and ADCP data. Deep-Sea Res., 48A, 269-295.

Grupo Gyroscope-España, 2002: Informe de la campaña Gyroscope E 03-2002. Datos y resúmenes del Instituto Español de Oceanografía, $25 \mathrm{pp}$.

Jenkins, G. M., and D. G. Watts, 1968: Spectral Analysis and Its Applications. Holden Day, $522 \mathrm{pp}$.

Lavín, A., 1999: Fluxes, trends and decadal changes in the subtropical North Atlantic. Ph.D. dissertation, Universidad de Cantabria, 222 pp.

- H. Bryden, and G. Parrilla, 2003: Mechanisms of heat, freshwater, oxygen and nutrient transports and budgets at $24.5^{\circ} \mathrm{N}$ in the subtropical North Atlantic. Deep-Sea Res., 50A, 1099 1128.
Lenschow, D. H., and B. B. Stankov, 1986: Length scales in the convective boundary layer. J. Atmos. Sci., 43, 1198-1209.

Maddox, R. A., 1980: An objective technique for separating macroscale and mesoscale features in meteorological data. Mon. Wea. Rev., 108, 1108-1121.

McGillicuddy, D. J., Jr., and A. R. Robinson, 1997: Eddy-induced nutrient supply and new production in the Sargasso Sea. Deep-Sea Res., 44A, 1427-1450.

Parrilla, G., A. Lavín, H. Bryden, M. García, and R. Millard, 1994: Rising temperatures in the subtropical North Atlantic Ocean over the past 35 years. Nature, $\mathbf{3 6 9}, 48-51$.

Pedder, M. A., 1989: Limited area kinematic analysis by a multivariate statistical interpolation method. Mon. Wea. Rev., 117, $1695-1708$.

Rodríguez, J., and Coauthors, 2001: Mesoscale vertical motion and the size structure of phytoplankton in the ocean. Nature, 410, 360-363.

Roemmich, D., 1983: Optimal estimation of hydrographic station data and derived fields. J. Phys. Oceanogr., 13, 1544-1549.

-, and C. Wunsch, 1984: Apparent changes in the climatic state of the deep North Atlantic Ocean. Nature, 307, 447-450.

_ and - 1985: Two transatlantic sections: Meridional circulation and heat flux in the subtropical North Atlantic Ocean. Deep-Sea Res., 32, 619-664.

Thiébaux, H. J., and M. A. Pedder, 1987: Spatial Objective Analysis: With Applications in Atmospheric Science. Academic Press, 300 pp.

Tintoré, J., D. Gomis, S. Alonso, and G. Parrilla, 1991: Mesoscale dynamics and vertical motion in the Alborán Sea. J. Phys. Oceanogr., 21, 811-823.

Wong, A. P. S., G. C. Johnson, and W. B. Owens, 2003: Delayedmode calibration of autonomous CTD profiling float salinity data by $\theta-S$ climatology. J. Atmos. Oceanic Technol., 20, 308318. 